Proceedings of the ASME 2017 International Design Engineering Technical Conferences \& Computers and Information in Engineering Conference

IDETC2017

August 6-9, 2017, Cleveland, Ohio, USA

IDETC2017-67791

\title{
EFFECT OF INITIAL CURVATURE ON THE STATIC AND DYNAMIC BEHAVIOR OF MEMS RESONATORS
}

\author{
Amal Z. Hajjaj \\ Physical Science and Engineering Division \\ King Abdullah University of Science and Technology \\ Thuwal, Makah Province, Saudi Arabia \\ amal.hajiaj@kaust.edu.sa
}

\author{
Nouha Alcheikh \\ Physical Science and Engineering Division \\ King Abdullah University of Science and Technology \\ Thuwal, Makah Province, Saudi Arabia \\ nouha.alcheikh@kaust.edu.sa
}

\author{
Mohammad I. Younis \\ Physical Science and Engineering Division \\ King Abdullah University of Science and Technology \\ Thuwal, Makah Province, Saudi Arabia \\ mohammad.younis@kaust.edu.sa
}

\begin{abstract}
In this paper, we investigate experimentally and analytically the effect of the initial shape, arc and cosine wave, on the static and dynamic behavior of microelectromechanical (MEMS) resonators. We show that by carefully choosing the geometrical parameters and the shape of curvature, the veering phenomenon (avoided-crossing) between the first two symmetric modes can be activated. To demonstrate this concept, we study electrothermally tuned and electrostatically driven MEMS initially curved resonators. Applying electrothermal voltage heats up the beams and then increases their curvature (stiffness) and controls their resonance frequencies. While changing the electrothermal voltage, we demonstrate high frequency tunability of arc resonators compared to the cosine-configuration resonators for the first and third resonance frequencies. For arc beams, we show that the first resonance frequency increases up to twice its fundamental value and the third resonance frequency decreases until getting very close to the first resonance frequency triggering the veering phenomenon. Around the veering regime, we study experimentally and analytically, using a reduced
\end{abstract}

order model based on a nonlinear Euler-Bernoulli shallow arch beam model, the dynamic behavior of the arc beam for different electrostatic forcing.

Keywords: Initially Curved Resonator, Electrothermal Actuation, Electrostatic Actuation, Veering Phenomenon.

\section{INTRODUCTION}

Microelectromechanical (MEMS) bistable structures have been the center of focus for several applications, such as mass/gas sensing [1], memory [2], logic [3], and filtering [4]. Bistable structures can be realized by intentional fabrication (arches) [3-4] or by tuning a control parameter, as in buckled structures [5]. Many studies have focused on investigating the static/dynamic behavior [6-8] of curved structures. Most of the arch beams studied in the literature assume an initial cosine-wave shape (similar to the buckled configuration) except for few studies that investigate curved strings of an arc shape [9-10]. In this work, we aim to explore the effect of initial shape and curvature on the static and dynamic behavior of initially curved beams, which are electrothermally tuned (to induce compressive loads) and electrostatically actuated. 
The static and dynamic behavior of arch beams under electrostatic actuation is well investigated in the literature [6-8]. At the micro scale, Ouakad and Younis [6] investigated the static and dynamic behavior of clamped-clamped micro-machined arches when actuated by a small DC electrostatic load superimposed to an AC harmonic load. At the nanoscale, many studies have investigated the static and dynamic behavior of clamped-clamped CNTs (slack). Sazonova et al. [8] reported experimental investigations showing the importance of slack on the dynamical behavior of CNTs.

Several actuation mechanisms have been used to study the effect of applying an axial load on the behavior of bistable structures, such as electrostatic [11], piezoresistive [12], electromagnetic [13], and electrothermal [3-4]. Many groups have used thermal actuation to investigate the static and dynamic buckling behavior of different bistable structures that are electrothermally actuated, such as U-shaped structures [14], V-shaped structures [15], and clamped-clamped structures [16]. Thermal actuation is also used to tune the resonance frequencies of bridge structures [3] through controlling their stiffness. The variation of the axial load, which accordingly changes the initial curvature of arch structures, can lead to the coupling among the different modes of vibration [4, 17]. In recent years, the modal coupling of vibration modes has been of intense interest. Those modes can be coupled mechanically [18], electrically [19], among the structure itself [20], and through internal resonances $[17,21]$. In a recent work [4], the coupling of the first two vibrational modes was shown experimentally for an initially curved beam electrothermally tuned for filter applications.

The veering phenomenon was firstly reported by Liessa [22]. It refers to the deviating of two Eigenfrequencies as varying a control parameter, here the electrothermal voltage, instead of continuing their path where they cross. Then, both modes get coupled, exchange energy and each one continues along the path that the other would have taken if they would cross. Few studies investigated the response of curved structures on the veering regime analytically [23-24]. Lacarbonara et al. [23] studied theoretically, using the multiple scales method, the nonlinear interaction among the modes of a hinged-hinged curved beam near veering.

In this paper, we aim to investigate the effect of the curvature on the variation of the resonance frequencies as well as the dynamic response of the system under consideration while applying an Ac and Dc electrostatic voltages and a constant electrothermal voltage. We aim to show the effect of the curvature on the activation of the veering phenomenon.

The rest of the paper is organized as follows. The nonlinear Euler-Bernoulli beam equation combined with the heat conduction equation is solved in Section 2. The experimental setup and results are presented in Section 3. A discussion of the variation of the eigenvalues is presented in Section 4. The dynamic response of curved beam under electrostatic forcing and at constant electrothermal load is reported in Section 5. Finally, the main conclusions are summarized in Section 6.

\section{PROBLEM FORMULATION}

The device under consideration consists of a clamped-clamped initially curved beam, presented in Fig. 1, of initial curvature $\hat{w}_{0}(\hat{x})$ governed by (1.a) for a cosine wave (buckled) shape and (1.b) for an arc shape:

$$
\begin{aligned}
& \hat{w}_{0}(\hat{x})=-\frac{1}{2} \hat{b}_{0}\left(1-\cos \left(2 \pi \frac{\hat{x}}{l}\right)\right) \\
& \hat{w}_{0}(\hat{x})=\left\{\begin{array}{l}
\left(\hat{b}_{0}-R+\sqrt{R^{2}-(\hat{x}-l / 2)^{2}}\right) \times \\
(u[1+\hat{x}]-u[\hat{x}])
\end{array}\right\}
\end{aligned}
$$

where $\hat{x}$ is the position along the microbeam, and $\hat{b}_{0}$ represents the rise at the mid-point of the arch. $u(\hat{x})$ and $R$ present the Heaviside function and the radius of the arc in (1.b), respectively. The curved beam has Young's modulus $E$, material density $\rho$, length $l$, width $b$, and thickness $h$. The cross section area of both configurations is assumed to be rectangular $A=$ $b h$ with a moment of inertia given by $I=b h^{3} / 12$. The curved beam is separated from a stationary electrode with a gap width $d$. The curved beam is actuated electrostatically by a DC polarization voltage $V_{D C}$ and an AC harmonic voltage of amplitude $V_{A C}$ and frequency $\hat{\Omega}$ and is subjected to a viscous damping of coefficient $\hat{c}$. The electrothermal voltage $V_{T H}$ is applied between the anchors of the curved beam inducing a current $I_{T H}$ passing through the beam that heats up it and controls its internally induced axial stress.

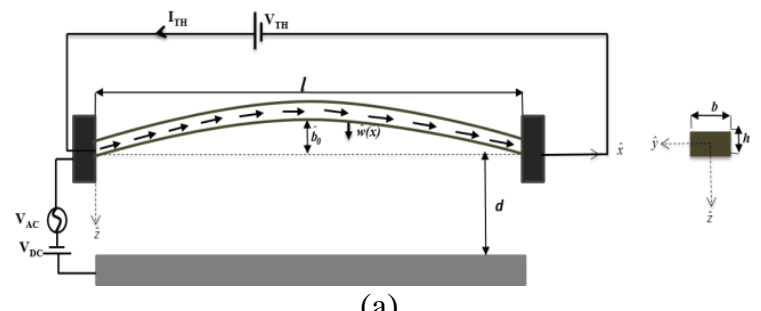

(a) 


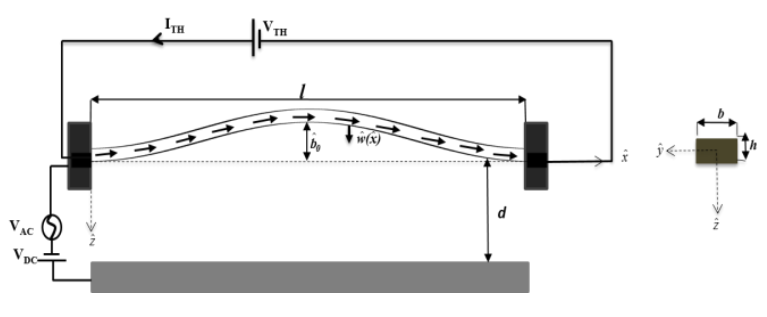

(b)

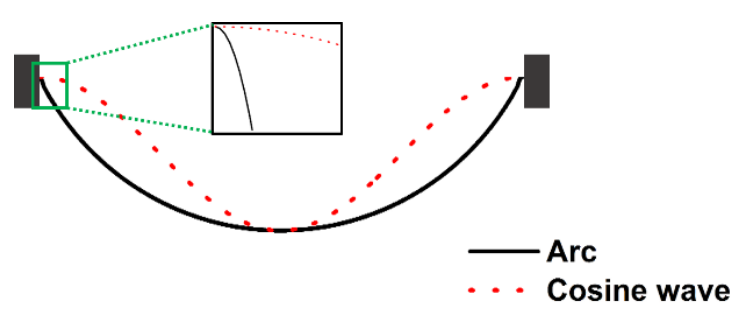

(c)

Figure 1: Schematic of an electrothermally actuated clampedclamped shallow arch (a) cosine wave (b) arc shape. (c) Schematic for both cosine wave and arc configurations highlighting their difference.

Referring to [3] and the Fourier's law, the equation governing the average temperature across the crosssection of the curved beam, induced by the current $I_{T H}$, is given by

$-\frac{d}{d \hat{x}}\left(k(T) \frac{d T}{d \hat{x}}\right)=J^{2} \rho_{e}(T)$

where $k(T)$ [25] is the thermal conductivity of the microbeam material, which has a nonlinear dependence on temperature. $\rho_{e}(T)$ denotes the electrical resistivity, which is assumed to have a linear dependence on temperature for the doped silicon [25]. The ends of the beam are assumed to be at room temperature $T_{a}$. The current density can be written as a function of the DC electrothermal voltage $J=V_{T H} / \rho_{e}(T) l$. Therefore, the Fourier's law equation can be written as

$-\frac{d}{d \hat{x}}\left(k(T) \frac{d T}{d \hat{x}}\right)=\frac{V_{T H}^{2}}{\rho_{e}(T) l^{2}}$

Thus, the variation of the temperature along the microbeam induces a compressive stress given by

$\hat{S}_{T H}=E A \int_{0}^{l} \frac{\alpha(T)\left(T[\hat{x}]-T_{a}\right)}{l} d \hat{x}$

where $\alpha(T)$ [25] is the coefficient of thermal expansion, which is assumed to be dependent on temperature.

The governing equation of motion of the curved beam under consideration, Fig. 1, describing its transverse deflection $\hat{w}(\hat{x}, \hat{t})$, where $\hat{t}$ is the time, is written as follows [6, 26]:

$$
\begin{aligned}
& \rho b h \frac{\partial^{2} \hat{w}}{\partial \hat{t}^{2}}+E I \frac{\partial^{4} \hat{w}}{\partial \hat{x}^{4}}+\hat{c} \frac{\partial \hat{w}}{\partial \hat{t}}= \\
& \left\{\begin{array}{l}
\left(\frac{\partial^{2} \hat{w}}{\partial \hat{x}^{2}}+\frac{d^{2} \hat{w}_{0}}{d \hat{x}^{2}}\right) \times \\
{\left[\hat{N}+\frac{E A}{2 l} \int_{0}^{l}\left(\left(\frac{\partial \hat{w}}{\partial \hat{x}}\right)^{2}+2 \frac{\partial \hat{w}}{\partial \hat{x}} \frac{d \hat{w}_{0}}{d \hat{x}}\right) d \hat{x}\right]}
\end{array}\right\} \\
& +\frac{1}{2} \varepsilon b \frac{\left(V_{D C}+V_{A C} \cos (\hat{\Omega} t)\right)^{2}}{\left(d-\hat{w}-\hat{w}_{0}\right)^{2}}
\end{aligned}
$$

The microbeam is subjected to the following boundary conditions:

$\hat{w}(0, \hat{t})=\hat{w}(l, \hat{t})=0$ and $\left.\frac{\partial \hat{w}}{\partial \hat{x}}\right|_{(0, \hat{t})}=\left.\frac{\partial \hat{w}}{\partial \hat{x}}\right|_{(l, \hat{t})}=0$

The term $\hat{N}=\hat{N}_{O}-\hat{S}_{T H}$ represents the axial load due to the residual axial load, where $\hat{N}_{O}$ is arising from the fabrication process and the compressive axial load and $\hat{S}_{T H}$ denotes the thermal compressive stress given by (4).

For convenience, we introduce the nondimensional variables as below:

$$
w=\frac{\hat{w}}{d} ; x=\frac{\hat{x}}{l} ; t=\frac{\hat{t}}{T} ; w_{0}=\frac{\hat{w}_{0}}{d} \text { and } b_{0}=\frac{\hat{b}_{0}}{d}
$$

where $T=\sqrt{\rho b h l^{4} / E I}$ is a time scale. Substituting (7) into (5) and (6), we obtain the nondimensional equation of motion of the beam

$$
\begin{aligned}
& \frac{\partial^{2} w}{\partial t^{2}}+\frac{\partial^{4} w}{\partial x^{4}}+c \frac{\partial w}{\partial t}= \\
& \left(\left(\frac{\partial^{2} w}{\partial x^{2}}+\frac{d^{2} w_{0}}{d x^{2}}\right)\left[N+\alpha_{1} \int_{0}^{1}\left[\frac{\partial w^{2}}{\partial x}+2 \frac{\partial w}{\partial x} \frac{d w_{0}}{d x}\right] d x\right]\right) \\
& +\alpha_{2} \frac{\left(V_{D C}+V_{A C} \cos (\Omega t)\right)^{2}}{\left(1-w-w_{0}\right)^{2}}
\end{aligned}
$$

subjected to the nondimensional boundary conditions

$$
w(0, t)=w(1, t)=0 \text { and }\left.\frac{\partial w}{\partial x}\right|_{(0, t)}=\left.\frac{\partial w}{\partial x}\right|_{(1, t)}=0
$$

The nondimensional parameters, except $\alpha_{2}$, appearing in (8) are defined as 
$\alpha_{1}=6 \frac{d^{2}}{h^{2}} ; \mathrm{N}=N_{0}+S_{T H} ; N_{0}=\frac{l^{2}}{E I} \hat{N}_{0}$

$; S_{T H}=\frac{l^{2}}{E I} \hat{S}_{T H} ; ; \alpha_{2}=\frac{6 \varepsilon l^{4}}{E h^{3} d^{3}} ;$

$c=\frac{l^{4}}{E I T} \hat{c}$ and $\Omega=T \hat{\Omega}$

\section{EXPERIMENTAL SETUP}

In this paper, the experimental validation was conducted on intentionally fabricated arc beams with specific initial curvatures. The arc beams were fabricated by MEMCAP [27], from SOI wafers with highly conductive Si device layer. To determine the resonance frequencies as well as the frequency response of such structures, we use a stroboscopic video microscope from Polytec [28], Fig. 2.

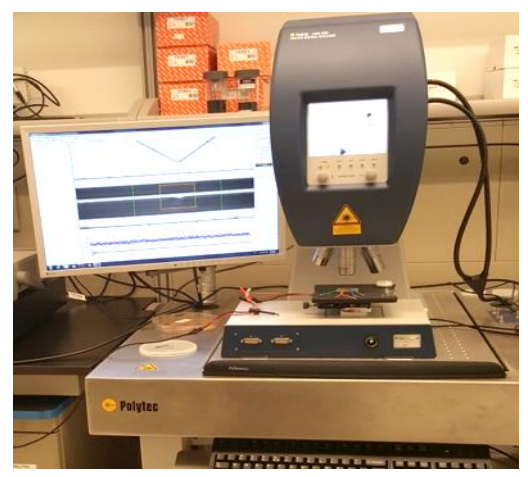

Figure 2: Experimental setup.

The arc beams are actuated electrothermally by passing a DC current, $I_{T H}$, through them, Fig. 3. Also, they can be actuated electrostatically to excite the structure into vibration. Three case studies will be investigated as shown in Table.

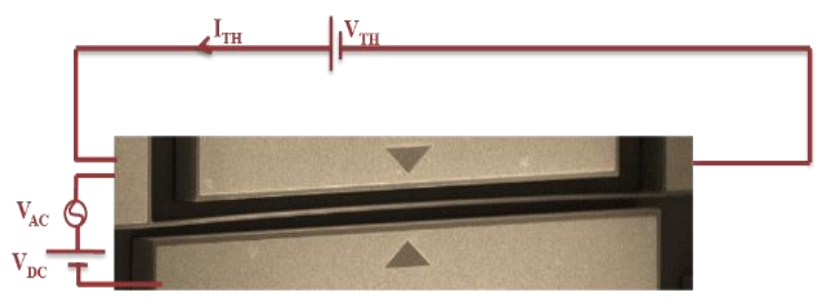

Figure 3: An SEM image of the arch beam electrothermally and electrostatically actuated.
TABLE 1. GEOMETRICAL PROPERTIES OF THE MICROBEAM MADE OF DOPED SILICON.

\begin{tabular}{|c|c|c|c|}
\hline Quantity & Arc Beam 1 & Arc Beam 2 & Arc Beam 3 \\
\hline Length $(\mu \mathrm{m})$ & 600 & 600 & 800 \\
\hline Thickness $(\mu \mathrm{m})$ & 2 & 2 & 2 \\
\hline Width $(\mu \mathrm{m})$ & 25 & 25 & 25 \\
\hline $\begin{array}{c}\text { Initial } \\
\text { curvature }(\mu \mathrm{m})\end{array}$ & 2 & 3 & 2.6 \\
\hline Gap $(\mu \mathrm{m})$ & 7 & 8 & 8 \\
\hline
\end{tabular}

\section{EIGENVALUE PROBLEM}

In this section, we examine the variation of the resonance frequencies of both curvature configurations, under consideration, as a function of the compressive load induced by the thermal voltage. Toward this, we drop the electrostatic force. Referring to [26], the deflection of the arch under the electrothermal force is split into a static deflection $w_{s}(x)$ due to $V_{T H}$ component and a small dynamic deflection $w_{d}(x, t)$.

The static equation is governed by

$$
\frac{d^{4} w_{s}}{d x^{4}}=\left(\begin{array}{l}
\left(\frac{d^{2} w_{s}}{d x^{2}}+\frac{d^{2} w_{0}}{d x^{2}}\right) \times \\
\left.\left[N+\alpha_{1} \int_{0}^{1}\left[\frac{d w_{s}^{2}}{d x}+2 \frac{d w_{s}}{d x} \frac{d w_{0}}{d x}\right] d x\right]\right)
\end{array}\right)
$$

with the associated boundary conditions

$$
w_{s}(0)=w_{s}(1)=0 \text { and }\left.\frac{d w_{s}}{d x}\right|_{x=0}=\left.\frac{d w_{s}}{d x}\right|_{x=1}=0
$$

The linearized equation of motion describing the small dynamic behavior of the curved beam around the new static configuration induced by $V_{T H}$ and governed by (11) is derived by substituting $w(x, t)=w_{s}(x)+w_{d}(x, t)$ into (5) and dropping the terms representing the equilibrium position, the electrostatic force, and the nonlinear terms. The outcome equation becomes

$$
\begin{aligned}
& \frac{\partial^{2} w_{d}}{\partial t^{2}}+\frac{\partial^{4} w_{d}}{\partial x^{4}}= \\
& {\left[N+\alpha_{1} \int_{0}^{1}\left[\frac{d w_{s}^{2}}{d x}+2 \frac{d w_{s}}{d x} \frac{d w_{0}}{d x}\right] d x\right] \frac{\partial^{2} w_{d}}{\partial x^{2}}} \\
& +2 \alpha_{1} \int_{0}^{I}\left[\left(\frac{d w_{s}}{d x}+\frac{d w_{0}}{d x}\right) \frac{\partial w_{d}}{\partial x}\right] d x\left(\frac{d^{2} w_{s}}{d x^{2}}+\frac{d^{2} w_{0}}{d x^{2}}\right)
\end{aligned}
$$


with the associated boundary conditions

$$
w_{d}(0, t)=w_{d}(1, t)=0 \text { and }\left.\frac{\partial w_{d}}{\partial x}\right|_{x=0, t}=\left.\frac{\partial w_{d}}{\partial x}\right|_{x=1, t}=0
$$

We resort to the Galerkin discretization to represent the dynamic deflection $w_{d}(x, t)$ and to solve the eigenvalue problem of the curved beam under the DC thermal voltage [26]. Toward this, we let

$$
w_{d}(x, t)=\sum_{i=0}^{n} u_{i}(t) \phi_{i}(x)
$$

Where $u_{i}(x)(i=0,1,2 . . n)$ denotes the nondimensional modal coordinates and $\phi_{i}(x)(i=0,1,2 . . n)$ denotes the mode shape of the unactuated straight clampedclamped beam.

Then, we substitute (15) into (13), multiplying the outcome by the mode shape $\phi_{j}$ and integrating over the beam domain (from 0 to 1 ), which yields the below equation [26]

$$
\begin{aligned}
& \ddot{u}_{j}=\left[2 \alpha_{1} \int_{0}^{1} \phi_{j} \frac{\partial^{2} w_{s}}{\partial x^{2}} d x \int_{0}^{1}\left(\sum_{i=0}^{n} u_{i} \phi_{i} \frac{\partial w_{s}}{\partial x}\right) d x\right] \\
& +\left[\alpha_{1} \int_{0}^{1}\left(\frac{\partial w_{s}}{\partial x}\right)^{2} d x\right] \int_{0}^{1} \phi_{j}\left(\sum_{i=0}^{n} u_{i} \phi_{i}^{\prime \prime}\right) d x
\end{aligned}
$$

Using five symmetric modes, we compute the Jacobin of the system of the five obtained algebraic equations, for each $V_{T H}$, and find the corresponding eigenvalues and mode shapes. Then, we compute the resonance frequencies of the resonators, at constant $V_{T H}$, by taking the square root of these eigenvalues.

Fig. 4 shows the variation of the first two symmetric resonance frequencies of the curved beams while tuning $V_{T H}$, experimentally and analytically for both configurations under consideration. One can note that for all case studies, the arc configuration shows a good agreement with the experimental results compared to the classically assumed cosine-wave (buckled) configuration. As shown in Figs. 4(a), 4(b) and $4(\mathrm{c})$, for the buckled configuration, the first resonance frequency increases while increasing the compressive load induced by $V_{T H}$. While tuning $V_{T H}$, the third resonance frequency decreases then starts to increase when the first resonance frequency seems to start to flatten.

On the other hand, and as proven experimentally, for the arc configuration, we reach higher tunability for both resonance frequencies. Indeed, the first resonance frequency increases as high as twice the fundamental frequency at zero electrothermal voltage. The third resonance frequency decreases and gets much closer to the first resonance frequency, and then they veer. Fig. 4(c) shows that for arc beam 3 the first and third resonance frequencies get very close at a critical electrothermal voltage. Then, they alter their directions, and each frequency continues along the path that the other frequency would have taken if they crossed, i.e., the first resonance frequency decreases while the third resonance frequency increases. This demonstrates the veering phenomenon (avoided-crossing). The veering phenomenon is a mechanical way to linearly couple the two involved modes.

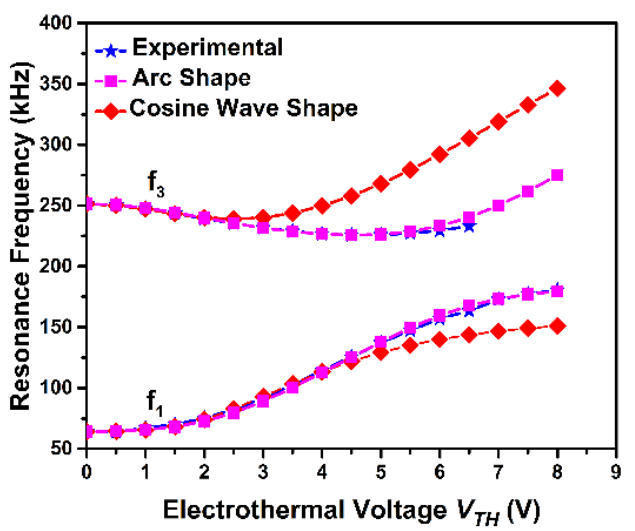

(a)

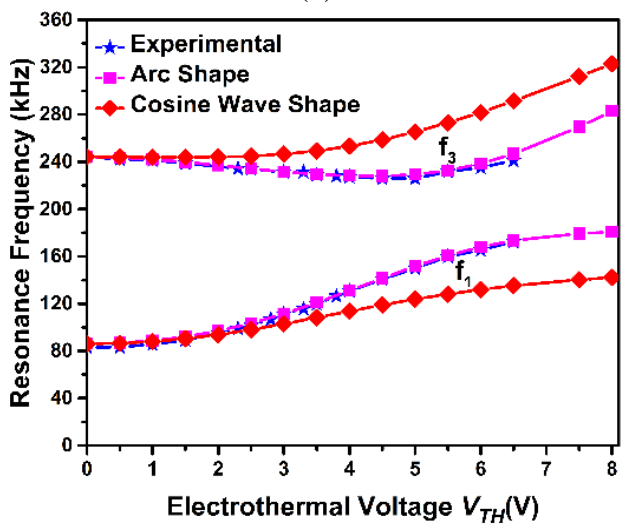

(b)

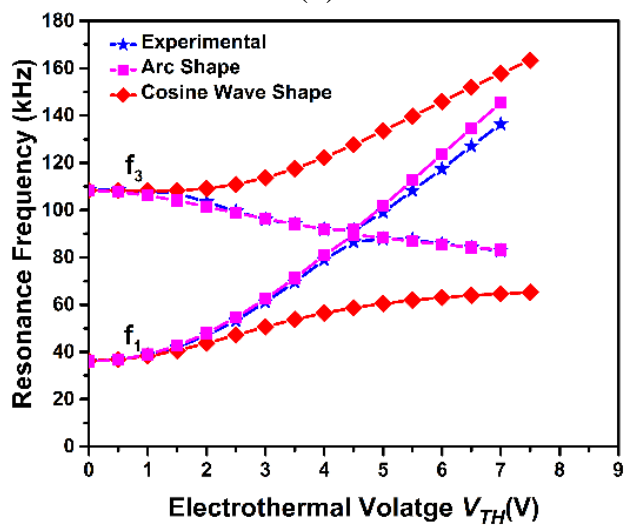

(c) 
Figure 4: The variation of the first two symmetric resonance frequencies while varying the electrothermal voltage of the curved beams for (a) Arc beam 1, (b) Arc beam 2, and (c) Arc beam 3.

To prove that for arc beam 3 we have an avoidedcrossing behavior, we analytically study the variation of the resonance frequency as varying the compressive load for the same beam but with different thicknesses, Fig. 5. Fig. 5(b) shows that for a specific thickness the third resonance decreases until getting very close to the first resonance frequency. Fig. 5 proves that we have an avoidedcrossing behavior. This presents a way to choose the geometric parameters of such curved beam carefully to activate or avoid veering phenomenon depending on the targeted application.

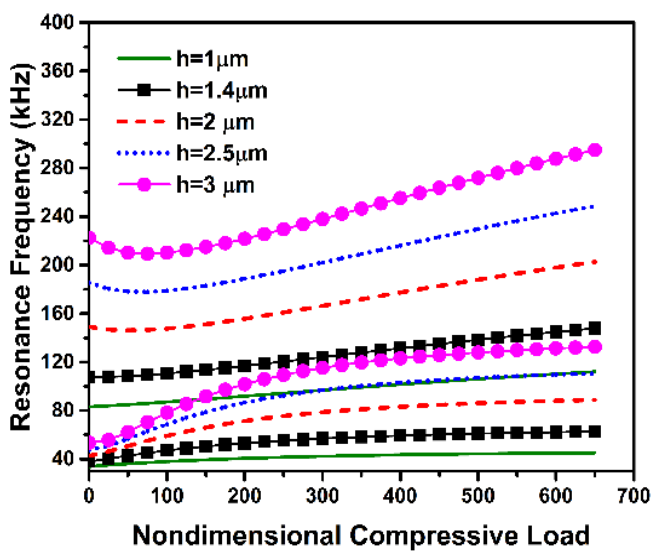

(a)

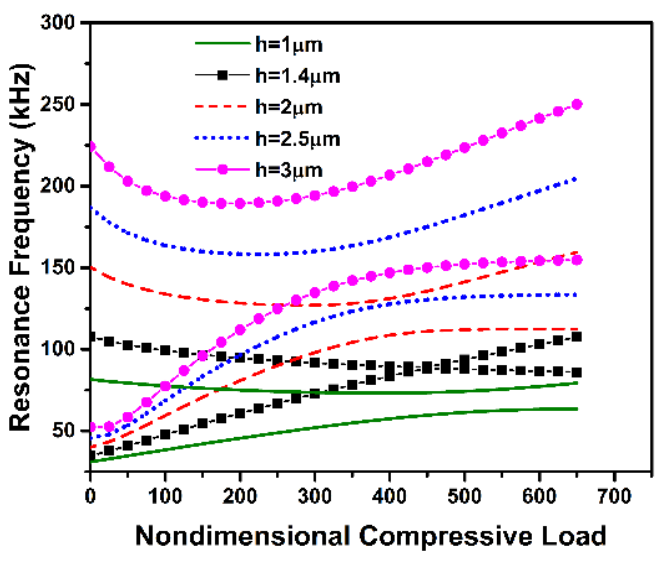

(b)

Figure 5: The variation of the first two symmetric frequencies of Arc beam 3 for different thicknesses and while changing the nondimensional compressive stress. (a) Cosine wave shape. (b) Arc shape.

For the arc beam 3, we investigate the variation of the different mode shapes around veering. By solving the mode shapes problem associated with (16). Table 2 shows the variation of the first three symmetric modes of arc beam 3 as varying the electrothermal voltage. One can note the interchange of mode shapes between the first and third vibrational modes at veering. This explains the increase in sensitivity, after veering, of the third mode compared to the first mode.

TABLE $2 . \quad$ FIRST THREE SYMMETRIC MODE SHAPES FOR VARIOUS ELECTROTHERMAL VOLTAGES

\begin{tabular}{|c|c|c|c|}
\hline$V_{T H}(V)$ & Mode 1 & Mode 3 & Mode 5 \\
\hline $\mathbf{0}$ & & & \\
\hline 3.5 & & & \\
\hline 4.25 & & & \\
\hline 4.5 & & & \\
\hline 4.625 & & & \\
\hline 4.75 & & & \\
\hline 5 & & & \\
\hline 6 & & & \\
\hline 7 & & & \\
\hline 8 & & & \\
\hline 9 & & & \\
\hline & & & \\
\hline
\end{tabular}

\section{DYNAMIC ANALYSIS}

Next, we further analyze the forced vibration response near the veering phenomenon of arc beam 3 . While exciting arc beam electrostatically, we study the dynamic response before, at, and after veering; i.e., for different values of electrothermal voltage.

Fig. 6 shows different frequency responses, experimentally obtained, of the arc beam for various electrothermal voltages and different excitation 
electrostatic voltages. Fig. 6(a) shows the dynamic response before veering at $V_{T H}=3.5 \mathrm{~V}$. The amplitude of vibration of the first mode is higher than the third mode, as expected since it is more sensitive than the third mode to the electrostatic forcing. As increasing the electrostatic voltage, the first and third modes exhibit softening and hardening behavior.

Getting much closer to the veering regime, $V_{T H}=4 \mathrm{~V}$, the amplitude of vibration of the third mode starts to increase comparing to the one of the first mode as increasing the electrostatic force, as shown in Fig. 6(b). This suggests that the third mode starts to take energy from the first mode. At veering, $V_{T H}=4.5 \mathrm{~V}$, both modes, first and third, start to exchange energy. Fig. 6(c) displays equal amplitude of vibration for both modes for different electrostatic voltages (i.e., same sensitivity to the electrostatic force for both modes).

Passing the veering zone, the third mode starts to be more sensitive than the first mode as shown in Fig. 6(d) $\left(V_{T H}=5 \mathrm{~V}\right)$ and Fig. 6(e) $\left(V_{T H}=6 \mathrm{~V}\right)$. Figs. 6(d) and 6(e) demonstrate that after veering the third mode takes the nonlinear properties of the first before veering. Indeed, it starts to exhibit softening behavior for high electrostatic forcing instead of hardening behavior. One can note that, for the same applied electrostatic force, the maximum amplitude of vibration at the third resonance frequency at $V_{T H}=5 \mathrm{~V}$ is higher than at $V_{T H}=4.5 \mathrm{~V}$, contrary to what is expected since the stiffness and the curvature increase more by increasing the electrothermal voltage. Figs. 6(d) and 6(e) demonstrate that the response of the first mode has weakened after veering.

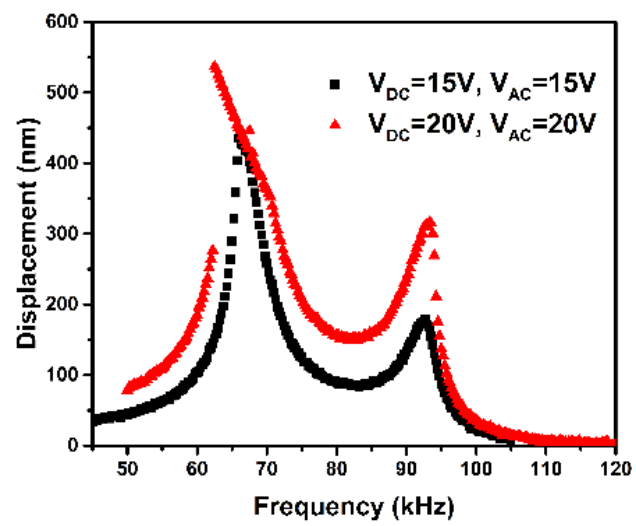

(a)

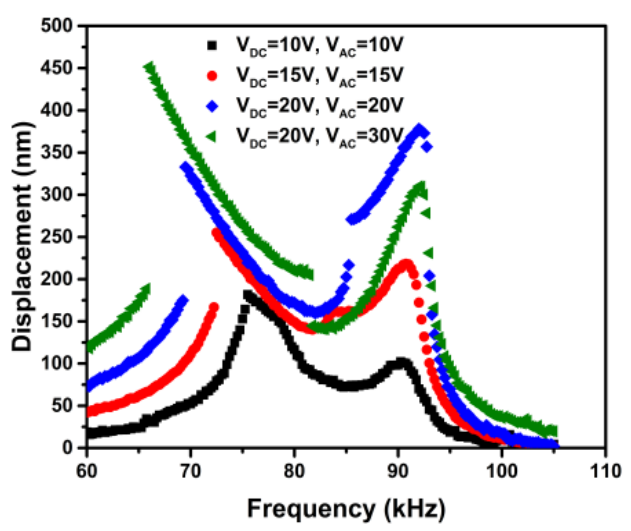

(b)

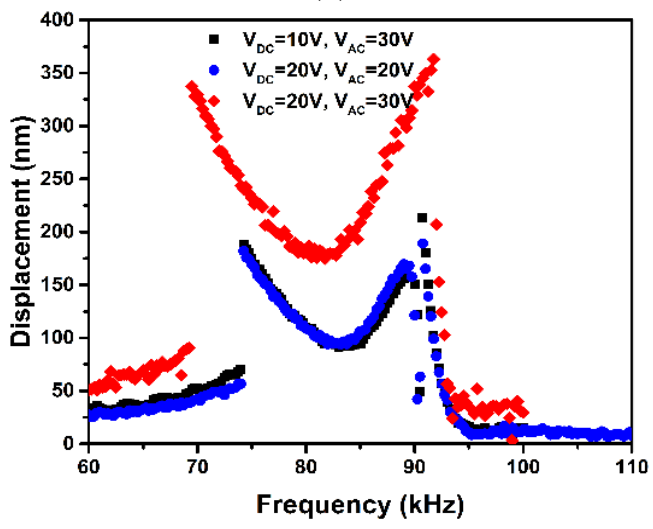

(c)

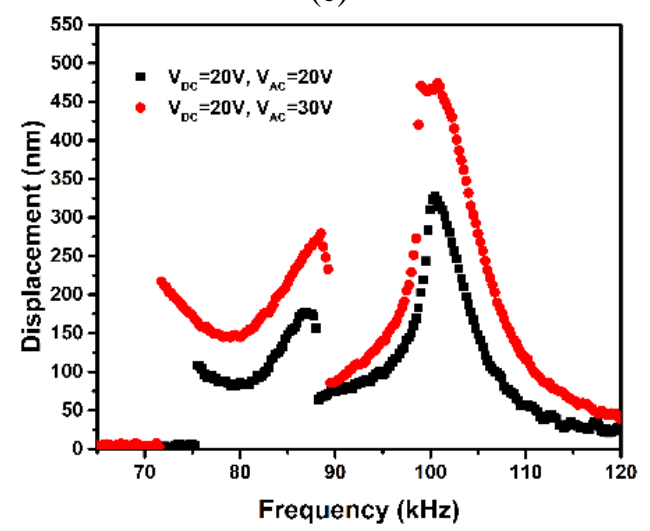

(d)

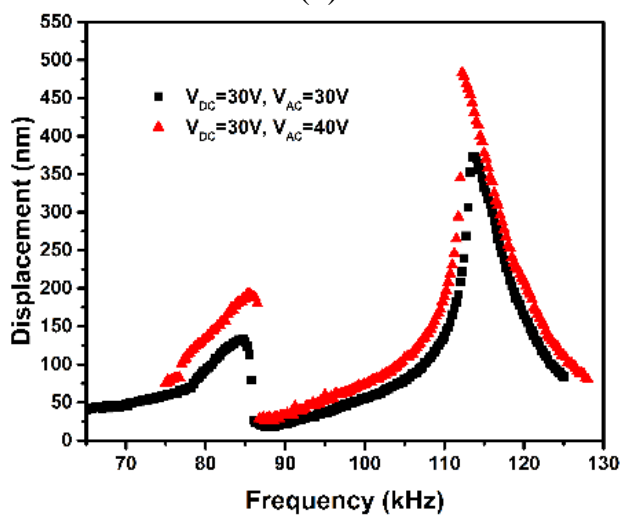

(e) 
Figure 6: Frequency response of arc beam (3) under different electrostatic force for different constant electrothermal voltages. (a) $V_{T H}=3.5 \mathrm{~V}$ (before veering), (b) $V_{T H}=4 \mathrm{~V}$ (close to veering), (c) $V_{T H}=4.5 \mathrm{~V}$ (on veering), (d) $V_{T H}=5 \mathrm{~V}$ (after veering) and (e) $V_{T H}=6 \mathrm{~V}$ (after veering).

To simulate the dynamic response of the arc beam under electrostatic forcing and at a constant electrothermal voltage, we discretize (8) using the Galerkin procedure, which yields a reduced order model (ROM). To do so, the transverse deflection of the arc beam is writing as follows [26]:

$$
w(x, t)=\sum_{i=0}^{n} q_{i}(t) \varphi_{i}(x)
$$

where $q_{i}(t)(i=0 \ldots n)$ are the nondimensional modal coordinates and $\varphi_{i}(x)(i=0 \ldots n)$ are the mode shapes obtained by solving the eigenvector problem associated with (16), at a constant electrothermal voltage.

Following [6,26], we first multiply (8) by $\left(1-w-w_{0}\right)^{2}$ in order to reduce the computational costs. Then, by substituting (17) in (8), multiplying by $\varphi_{j}(x)$ and integrating along the arc beam, this yields to $\mathrm{n}$ algebraic equation in terms of $q_{i}(t)$

$$
\begin{aligned}
\sum_{i=0}^{n} M_{i j} \ddot{q}_{i}(t) & +\sum_{i=0}^{n} c_{i j} \dot{q}_{i}(t)+\sum_{i=0}^{n} K_{i j} q_{i}(t) \\
& =F m_{j}+F e_{j}(t)
\end{aligned}
$$

$\forall(j=0 . . . n)$

where

$$
\begin{aligned}
& M_{i j}=\int_{0}^{1}\left(\varphi_{i}(x) \varphi_{j}(x)\left(1-w(x)-w_{0}(x)\right)^{2}\right) d x \\
& c_{i j}=c \int_{0}^{1}\left(\varphi_{i}(x) \varphi_{j}(x)\left(1-w(x)-w_{0}(x)\right)^{2}\right) d x \\
& K_{i j}=\int_{0}^{1}\left(\varphi_{i}^{(i v)}(x) \varphi_{j}(x)\left(1-w(x)-w_{0}(x)\right)^{2}\right) d x
\end{aligned}
$$$$
F e_{j}(t)=\alpha_{2}\left(V_{D C}+V_{A C} \operatorname{Cos}(\Omega t)\right)^{2} \int_{0}^{1} \varphi_{j}(x) d x
$$$$
F m_{j}=\alpha_{1} \Gamma^{*}
$$$$
\int_{0}^{1}\left(\begin{array}{l}
\varphi_{j}(x)\left(\sum_{i=0}^{n} q_{i}(t) \varphi_{i}{ }^{\prime \prime}(x)+w_{0}{ }^{\prime \prime}(x)\right) * \\
\left(1-w(x)-w_{0}(x)\right)^{2} d x
\end{array}\right)
$$

$$
\begin{aligned}
\Gamma= & N+\int_{0}^{l}\left(\sum_{i=0}^{n} q_{i}(t) \varphi_{i}{ }^{\prime}(x)\right)^{2} d x \\
& +2 \int_{0}^{l}\left(\sum_{i=0}^{n} q_{i}(t) \varphi_{i}{ }^{\prime}(x)\right) w_{0}{ }^{\prime}(x) d x
\end{aligned}
$$

Using Runge-Kutta technique, the dynamic response of the arc beam is obtained by time-integrating (18). Using three mode shapes, Figs. 7(a) and 7(b) a good agreement is reported between the simulations and the experimental results for $V_{T H}=3.5 \mathrm{~V} \quad\left(V_{D C}=15 \mathrm{~V}\right.$, $\left.V_{A C}=15 \mathrm{~V}\right)$ and $V_{T H}=4 \mathrm{~V} \quad\left(V_{D C}=10 \mathrm{~V}, \quad V_{A C}=10 \mathrm{~V}\right)$, respectively. For $V_{T H}=4 \mathrm{~V}$, increasing more the electrostatic force we start to have a mismatching between the experimental and the analytical. On the other hand, the ROM was able to detect the response of the system around the third mode accurately, Figs. $7(\mathrm{c})$ and 7(d). Increasing more the electrothermal voltage $V_{T H}=5 \mathrm{~V}$ (after veering regime), the ROM was able to detect the change of nonlinear behavior of the third mode from hardening to softening behavior while failing to accurately predict the response around the first mode.

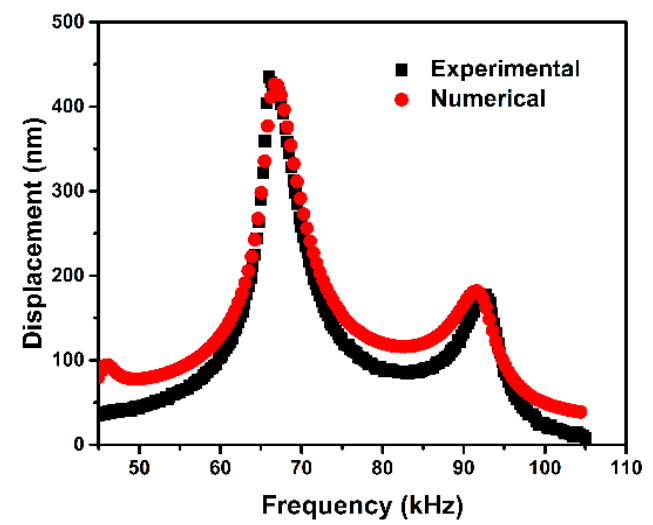

(a)

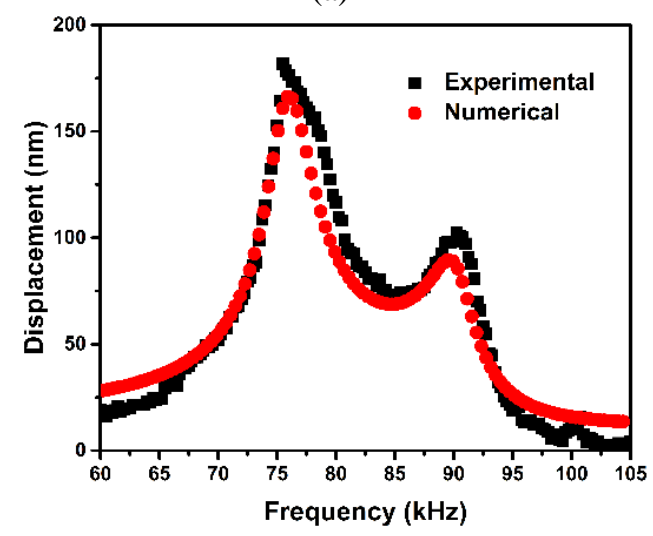

(b) 


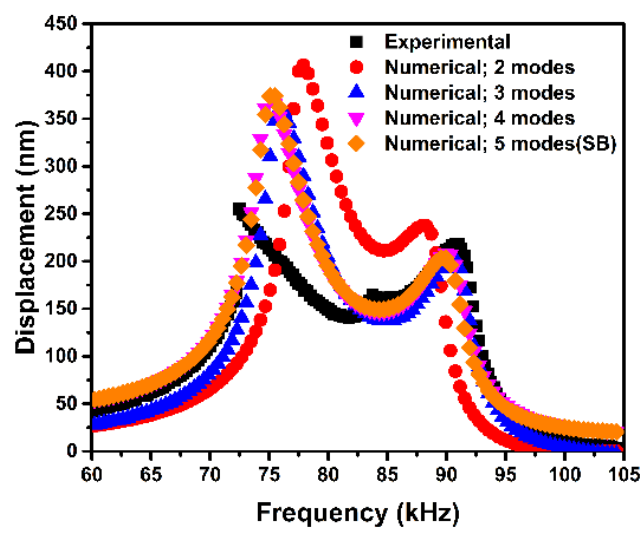

(c)

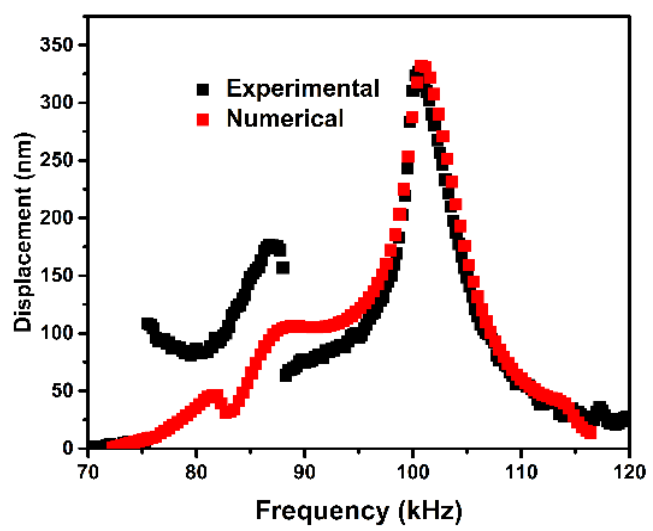

(d)

Figure 7: Analytical and experimental frequency response curves of arc beam (3) under electrostatic force and different constant electrothermal voltages. (a) $V_{T H}=3.5 \mathrm{~V}, V_{D C}=15 \mathrm{~V}$ and $V_{A C}=15 \mathrm{~V}$, (b) $V_{T H}=4 \mathrm{~V}, V_{D C}=10 \mathrm{~V}$ and $V_{A C}=10 \mathrm{~V}$, (c) $V_{T H}=4 \quad \mathrm{~V}, V_{D C}=15 \mathrm{~V}$ and $V_{A C}=15 \mathrm{~V}$, (d) $V_{T H}=5 \mathrm{~V}, V_{D C}=20 \mathrm{~V}$ and $V_{A C}=20 \mathrm{~V}$.

One can note that the ROM could not completely capture the dynamic behavior near and after veering. One reason might be that the ROM does not take into consideration the contribution of the out-of-plane and rotational modes in the response, which experimentally is observed to affect the response. We have noticed high sensitivity of such modes as increasing the electrothermal voltage when doing the experiments. Such modes could be easily seen and distinguished during the experiment using the high speed camera. Other techniques can be employed to demonstrate theoretically the veering phenomenon, mainly the multiple scale methods [23] that would be considered in future work.

\section{CONCLUSIONS}

In this paper, we showed that by choosing carefully the geometric parameters of an initially curved arc beam, we could activate the veering phenomenon. The initially curved beams considered in this work are electrothermally tuned and electrostatically actuated. High tunability for the first two symmetric resonance frequencies is showen as varying the compressive load induced by the applied electrothermal voltage. We studied theoretically, using the reduced order model, and experimentally the dynamic behavior of such resonator before, at, and after veering. In veering regime, the first and third modes exchange energy. After veering, we demonstrated that the third mode takes the nonlinear behavior of the first mode before veering and starts to be more sensitive than the first mode. We showed that the ROM can capture most of the dynamical behavior; however suffers near the veering regime. Other methods, such as the multiple scales, can be used to further study the dynamics near the veering regime.

\section{REFERENCES}

[1] Bouchaala, A., Jaber, N., Shekhah, O., Chernikova, V., Eddaoudi, M., and Younis, M. I., 2016, "A smart microelectromechanical sensor and switch triggered by gas," Applied Physics Letters, 109(1), p. 013502.

[2] Charlot, B., Sun, W., Yamashita, K., Fujita, H., and Toshiyoshi, H., 2008, "Bistable nanowire for micromechanical memory," Journal of Micromechanics and Microengineering, 18(4), p. 045005 .

[3] Hafiz, M., Kosuru, L., and Younis, M. I., 2016, "Microelectromechanical reprogrammable logic device," Nature communications, 7.

[4] Hajjaj, A. Z., Hafiz, M. A., and Younis, M. I., 2017, "Mode Coupling and Nonlinear Resonances of MEMS Arch Resonators for Bandpass Filters," Scientific Reports, 7, p. 41820.

[5] Hajjaj, A. Z., Alcheikh, N., Ramini, A., Al Hafiz, M. A., and Younis, M. I., 2016, "Highly tunable electrothermally and electrostatically actuated resonators," Journal of Microelectromechanical Systems, 25(3), pp. 440-449.

[6] Ouakad, H. M., and Younis, M. I., 2010, "The dynamic behavior of MEMS arch resonators actuated electrically," International Journal of Non-Linear Mechanics, 45(7), pp. 704-713.

[7] Krylov, S., Ilic, B. R., Schreiber, D., Seretensky, S., and Craighead, H., 2008, "The pull-in behavior of electrostatically actuated bistable microstructures," Journal of Micromechanics and Microengineering, 18(5), p. 055026.

[8] Sazonova, V., Yaish, Y., Üstünel, H., Roundy, D., Arias, T. A., and McEuen, P. L., 2004, "A tunable

Copyright $\odot 2017$ by ASME 
carbon nanotube electromechanical oscillator," Nature, 431(7006), pp. 284-287.

[9] Lacarbonara, W., Paolone, A., and Vestroni, F., 2007, "Elastodynamics of nonshallow suspended cables: linear modal properties," Journal of Vibration and Acoustics, 129(4), pp. 425-433.

[10] Lacarbonara, W., and Rega, G., 2003, "Resonant non-linear normal modes. Part II: activation/orthogonality conditions for shallow structural systems," International Journal of NonLinear Mechanics, 38(6), pp. 873-887.

[11] Alcheikh, N., Ramini, A., Hafiz, M. A. A., and Younis, M. I., 2017, "Tunable Clamped-Guided Arch Resonators Using Electrostatically Induced Axial Loads," Micromachines, 8(1), p. 14.

[12] Svilicic, B., Mastropaolo, E., Flynn, B., and Cheung, R., 2012, "Electrothermally actuated and piezoelectrically sensed silicon carbide tunable MEMS resonator," IEEE electron device letters, 33(2), pp. 278-280.

[13] Cottone, F., Basset, P., Vocca, H., Gammaitoni, L., and Bourouina, T., 2014, "Bistable electromagnetic generator based on buckled beams for vibration energy harvesting," Journal of Intelligent Material Systems and Structures, 25(12), pp. 1484-1495.

[14] Lerch, P., Slimane, C. K., Romanowicz, B., and Renaud, P., 1996, "Modelization and characterization of asymmetrical thermal micro-actuators," Journal of Micromechanics and Microengineering, 6(1), p. 134.

[15] Maloney, J. M., Schreiber, D. S., and DeVoe, D. L., 2003, "Large-force electrothermal linear micromotors," Journal of Micromechanics and Microengineering, 14(2), p. 226.

[16] Chen, X., Ma, L., Zheng, Y., and Lee, D.-W., 2011, "Theoretical analysis of postbuckling behavior with experimental validation using electrothermal microbeams," Applied Physics Letters, 98(7), p. 073107.

[17] Ramini, A. H., Hajjaj, A. Z., and Younis, M. I., 2016, "Tunable Resonators for Nonlinear Modal Interactions," Scientific Reports, 6.

[18] Karabalin, R., Cross, M., and Roukes, M., 2009, "Nonlinear dynamics and chaos in two coupled nanomechanical resonators," Physical Review B, 79(16), p. 165309.

[19] Lan, C., Qin, W., and Deng, W., 2015, "Energy harvesting by dynamic unstability and internal resonance for piezoelectric beam," Applied Physics Letters, 107(9), p. 093902.

[20] Venstra, W. J., van Leeuwen, R., and van der Zant, H. S., 2012, "Strongly coupled modes in a weakly driven micromechanical resonator," Applied Physics Letters, 101(24), p. 243111.

[21] Antonio, D., Zanette, D. H., and López, D., 2012, "Frequency stabilization in nonlinear micromechanical oscillators," Nature communications, 3, p. 806.

[22] Leissa, A. W., 1974, "On a curve veering aberration," Zeitschrift für angewandte Mathematik und Physik ZAMP, 25(1), pp. 99-111.

[23] Lacarbonara, W., Arafat, H. N., and Nayfeh, A. H., 2005, "Non-linear interactions in imperfect beams at veering," International Journal of Non-Linear Mechanics, 40(7), pp. 987-1003.

[24] Ouakad, H. M., and Younis, M. I., 2011, "Natural frequencies and mode shapes of initially curved carbon nanotube resonators under electric excitation," Journal of Sound and Vibration, 330(13), pp. 3182-3195.

[25] Lott, C. D., McLain, T. W., Harb, J. N., and Howell, L. L., 2002, "Modeling the thermal behavior of a surface-micromachined linear-displacement thermomechanical microactuator," Sensors and Actuators A: Physical, 101(1), pp. 239-250.

[26] Younis, M. I., 2011, MEMS linear and nonlinear statics and dynamics, Springer Science \& Business Media.

[27][Online].MEMSCAP:http://www.memscap.com/. [28] [Online].Polytech: http://www.polytec.com/us/. 